\section{P38 inhibition in COPD; cautious optimism}

\author{
Dave Singh
}

The nature of inflammation varies greatly between COPD patients, leading to heterogeneity in clinical manifestations. For example, emphysema is severe in some patients but minimal in others. ${ }^{1}$ The varied nature of COPD inflammation means that a novel anti-inflammatory drug is unlikely to be effective in all patients. This is certainly true for established anti-inflammatory therapies such as inhaled corticosteroids, which are most effective in the subgroup of patients with a history of exacerbations, ${ }^{2}$ and also those with evidence of increased sputum eosinophils. ${ }^{3}$ The PDE4 inhibitor, roflumilast, also shows greater efficacy in a COPD subgroup; those with chronic bronchitis and a history of exacerbations. ${ }^{4}$

The p38 mitogen activated protein kinase (MAPK) signalling pathway promotes inflammation by enhancing inflammatory gene transcription, stabilising mRNAs and increasing protein translation. $^{5}$ p38 MAPK signalling is activated by different extracellular stimuli that are relevant to COPD, such as cytokines, tolllike receptor agonists and oxidative stress including components of cigarette smoke. ${ }^{5-7}$ Furthermore, lung immunohistochemistry studies have demonstrated increased p38 MAPK activation in COPD patients compared with healthy controls. $^{8} 9$ p38 MAPK inhibitors reduce cytokine production from different COPD lung cells in vitro, including alveolar macrophages, ${ }^{6}$ lymphocytes and bronchial epithelial cells. ${ }^{8}$ These observations from laboratory studies indicate that p38 MAPK activation contributes to COPD inflammation, and have encouraged the development of p38 MAPK inhibitors to treat COPD. ${ }^{10} 11$

Orally administered p38 MAPK inhibitors have been developed for the treatment of inflammatory diseases including rheumatoid arthritis. ${ }^{12} 13$ Unfortunately, these drugs have caused unacceptable side effects including liver toxicity and skin reactions. Additionally, the efficacy appears to be transient; initial improvements in systemic inflammatory biomarkers including C-reactive protein (CRP)

Correspondence to Prof Dave Singh, University Of Manchester, Medicines Evaluation Unit, Manchester M23 9QZ, UK; dsingh@meu.org.uk disappear within weeks. ${ }^{12} 13$ This may be due to 'redundancy', where blocking one pathway within a complex inflammation network simply diverts the signal to activate a different pathway. p38 MAPK inhibition may result in upstream kinases redirecting signalling through other MAPKs, such as extracellular signalrelated kinase (ERK), and c-Jun N-terminal kinase (JNK) to propagate inflammation. ${ }^{13}$ We hope that the transient efficacy observed in rheumatoid arthritis patients does not occur in COPD clinical trials of p38 MAPK inhibitors. The mechanisms of inflammation differ between these two diseases, and activation of redundant MAPKs after p38 MAPK inhibition may not occur in COPD. Nevertheless, treating inflammatory diseases using highly selective p38 MAPK inhibitors may be an approach that is too focused; using drugs that also target a wider spectrum of inflammatory kinases may overcome redundancy. ${ }^{13}$ Furthermore, most p38 MAPK inhibitors, including the novel inhibitor $\mathrm{PH}-797804,{ }^{14}$ selectively target the $\alpha$ isoform which is highly expressed on immune cells. However, the other isoforms ( $\beta, \gamma$ and $\delta$ ) may also be important in inflammation, and certainly are expressed in COPD lung tissue. ${ }^{8} 15$ Broader targeting of p38 MAPK isoforms may be more effective in COPD.

Macnee et al report a 6-week parallel group, placebo controlled, randomised clinical trial in COPD patients of the orally administered p38 MAPK inhibitor PH-797804. ${ }^{16}$ The dose-response effects of $0.5,3,6$ and $10 \mathrm{mg}$ once daily were evaluated, with more patients receiving $6 \mathrm{mg}$, as this dose was anticipated to have the optimum therapeutic index. An interim futility analysis resulted in no further patients being recruited to receive the $0.5 \mathrm{mg}$ dose as there was $<10 \%$ chance of achieving $\geq 75 \mathrm{ml}$ improvement over placebo in $\mathrm{FEV}_{1}$ change from baseline. The final randomisation included more patients who received $6 \mathrm{mg}(\mathrm{n}=70)$, compared with the remaining treatment arms $(n=45$ to 48$)$. The $6 \mathrm{mg}$ dose had the greatest clinical efficacy, demonstrating a statistically significant $93 \mathrm{ml}$ improvement in FEV1 compared with placebo associated with an improvement in breathlessness measured using the TDI (0.99, $\mathrm{p}=0.049$ ), and also significant improvements in inspiratory capacity and rescue medication use. The lung function improvements coupled with symptomatic benefits is convincing evidence of a therapeutic effect. However, this study had a limited sample size, and there were wide 95\% CIs from 18 to $166 \mathrm{ml}$ for the FEV1 change caused by PH-797804 6 mg compared with placebo. The true effect of this drug will only become apparent in larger studies. PH-797804 treatment was associated with some side effects, notably a rash, but was generally well tolerated.

The effects of PH-797804 $3 \mathrm{mg}$ and $10 \mathrm{mg}$ were smaller than the $6 \mathrm{mg}$ dose. PH-797804 $10 \mathrm{mg}$ caused a nonsignificant $66 \mathrm{ml}$ improvement in FEV1 compared with placebo, while the TDI score and rescue medication use were also not different to placebo. The reason for the apparent effectiveness of $6 \mathrm{mg}$ but not $10 \mathrm{mg}$ is unclear. Let us speculate. In rheumatoid arthritis, p38 MAPK inhibition may lose effectiveness due to redundant mechanisms; is it possible that the degree of p38 MAPK inhibition caused by the highest $\mathrm{PH}-797804$ dose activated other MAPKs that consequently negated any therapeutic effect? A counter argument is that doses from 3 to $10 \mathrm{mg}$ suppressed CRP levels at 6 weeks, indicating no loss of effect of the $10 \mathrm{mg}$ dose in the systemic circulation. A different reason for this result could be that a smaller sample size received $10 \mathrm{mg}$ compared with $6 \mathrm{mg}$, reducing the statistical power to detect drug effects at the higher dose. Nevertheless, this study has reported an apparently bell-shaped dose response curve, and understanding the reason for this may be very important for advancing our understanding of the p38 MAPK pathway in COPD.

The patients recruited for this study of PH-797804 were not restricted to a particular phenotype, but were simply moderate to severe COPD patients. ${ }^{16} \mathrm{PH}-797804$ might be most effective in a subgroup of COPD patients with inflammation that is highly dependent on P38 MAPK activation. Do we have any clues as to the clinical characteristics of this phenotype? The oral p38 MAPK inhibitor losmapimod did not change FEV1 over 12 weeks in COPD patients, but a cluster analysis showed an improvement in hyperinflation measurements. ${ }^{11}$ PH-797804 also improved inspiratory capacity. ${ }^{16}$ p38 MAPK activation is increased in the small airways of COPD patients, ${ }^{8}{ }^{9}$ so perhaps p38 MAPK inhibitors reduce small airway inflammation, thereby reducing air trapping in patients with hyperinflation. Alternatively, 
it has been reported that quadriceps inflammation in COPD patients is associated with increased p38 MAPK activation, ${ }^{17}$ although another study failed to replicate this finding. ${ }^{18}$ PH-797804 and losmapimod both reduced systemic biomarkers of inflammation in COPD patients, ${ }^{11} 16$ suggesting that these drugs have systemic activity which may target p38 MAPK-driven muscle inflammation.

Losmapimod failed to show an effect on the primary endpoint of sputum neutrophils in COPD patients. ${ }^{11}$ The effects of $\mathrm{PH}-797804$ on airway inflammation were not measured. ${ }^{16}$ Measuring inflammation in COPD clinical trials can be difficult, as sputum studies can be prone to practical difficulties and variability, while bronchoscopy studies are invasive. One assumes that the improvement in FEV1 caused by $\mathrm{PH}-797804$ was due to an antiinflammatory effect on lung cells. However, oral p38 MAPK inhibitors may also exert therapeutic benefits by targeting systemic inflammation in COPD patients, ${ }^{10} 11$ and the extent to which these drugs inhibit pulmonary inflammation is currently unknown. Oral p38 MAPK inhibitors often have systemic side effects, and a possible future avenue for p38 MAPK inhibitors is delivery by inhalation to minimise systemic exposure and, hence, adverse effects. However, this strategy might backfire, as inhaled delivery may also reduce therapeutic benefits derived from systemic exposure.

Macnee et al studied PH-797804 in patients who were not taking inhaled corticosteroids or long-acting bronchodilators. $^{16}$ Understanding the effects of PH-797804 in such patients is important, as this may reflect the potential use of $\mathrm{PH}-797804$ in clinical practice. Furthermore, there are interesting in vitro data concerning p38 MAPK inhibitors and corticosteroids that could be important in the clinic. p38 MAPK activation is corticosteroid-resistant in COPD alveolar macrophages. $^{6} \quad$ PH-797804 should provide an additive anti-inflammatory effect when used with a corticosteroid by inhibiting this corticosteroid-resistant mechanism. Moreover, p38 MAPK inhibitors and corticosteroids used in combination cause more than additive suppression of cytokine production from COPD alveolar macrophages; ${ }^{6}$ this synergism can be explained by molecular interactions between the p38 MAPK and glucocorticoid receptor signalling pathways. ${ }^{19}$ It would be a huge therapeutic breakthrough if such an effect was also observed in clinical trials.

Macnee et al are the first investigators to describe an improvement in FEV1 caused by a p38 MAPK inhibitor in COPD patients. ${ }^{16}$ This is an encouraging beginning. The study duration was 6 weeks; longer studies are needed to investigate whether the transient efficacy of p38 MAPK inhibitors in rheumatoid arthritis also occurs in COPD. The development of novel anti-inflammatory drugs for COPD has been notoriously difficult, as it is necessary to understand the likely 'responder' populations. The clinical phenotype most likely to respond to PH-797804 is currently unknown, and needs to be defined for the drug to stand the best chance of being successfully developed. There are other important questions about $\mathrm{PH}-797804$ that also need answering; how well will it work when given with inhaled corticosteroids? Will the side-effect profile be acceptable? Rheumatologists have grown pessimistic about the potential of p38 MAPK inhibitors. We can be cautiously optimistic about PH-797804 in COPD, but there are still considerable challenges ahead.

To cite Singh D. Thorax 2013;68:705-706.

Published Online First 23 April 2013

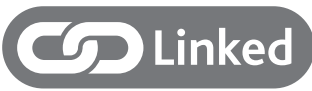

- http://dx.doi.org/10.1136/thoraxjnl-2012-202744

Thorax 2013:68:705-706

doi:10.1136/thoraxjn-2013-203498

\section{REFERENCES}

1 Agusti A, Calverley PM, Celli B, et al. Characterisation of COPD heterogeneity in the ECLIPSE cohort. Respir Res 2010;11:122.

2 Calverley PM, Anderson JA, Celli B, et al. Salmeterol and fluticasone propionate and survival in chronic obstructive pulmonary disease. N Engl I Med 2007:356:775-89.

3 Brightling $C E$, Monteiro W, Ward R, et al. Sputum eosinophilia and short-term response to prednisolone in chronic obstructive pulmonary disease: a randomised controlled trial. Lancet 2000:356:1480-5.

4 Rennard SI, Calverley PM, Goehring UM, et al. Reduction of exacerbations by the PDE4 inhibitor roflumilast-the importance of defining different subsets of patients with COPD. Respir Res 2011;12:18

5 Saklatvala J. The p38 MAP kinase pathway as a therapeutic target in inflammatory disease. Curr Opin Pharmacol 2004:4:372-7.

6 Armstrong J, Harbron C, Lea S, et al. Synergistic effects of p38 mitogen-activated protein kinase inhibition with a corticosteroid in alveolar macrophages from patients with chronic obstructive pulmonary disease. I Pharmacol Exp Ther 2011;338:732-40.

7 Moretto N, Bertolini S, ladicicco C, et al. Cigarette smoke and its component acrolein augment IL-8/ CXCL8 mRNA stability via p38 MAPK/MK2 signaling in human pulmonary cells. Am J Physiol Lung Cell Mol Physiol 2012;303:L929-38.

8 Gaffey K, Reynolds S, Plumb J, et al. Increased phosphorylated p38 mitogen activated protein kinase in COPD lungs. Eur Respir J Published Online First: 11 October 2012. doi:10.1183/09031936.00170711

9 Renda T, Baraldo S, Pelaia G, et al. Increased activation of p38 MAPK in COPD. Eur Respir $J$ 2008:31:62-9.

10 Singh D, Smyth L, Borrill Z, et al. A randomized, placebo-controlled study of the effects of the p38 MAPK inhibitor SB-681323 on blood biomarkers of inflammation in COPD patients. J Clin Pharmacol 2010:50:94-100.

11 Lomas DA, Lipson DA, Miller BE, et al. An oral inhibitor of p38 MAP kinase reduces plasma fibrinogen in patients with chronic obstructive pulmonary disease. J Clin Pharmacol 2012;52:416-24.

12 Genovese MC, Cohen SB, Wofsy D, et al. A 24-week, randomized, double-blind, placebo-controlled, parallel group study of the efficacy of oral SCIO-469, a p38 mitogen-activated protein kinase inhibitor, in patients with active rheumatoid arthritis. I Rheumatol 2011:38:846-54.

13 Hammaker D, Firestein GS. "Go upstream, young man": lessons learned from the p38 saga. Ann Rheum Dis 2010;69:i77-82.

14 Hope HR, Anderson GD, Burnette BL, et al. Anti-inflammatory properties of a novel $\mathrm{N}$-phenyl pyridinone inhibitor of p38 mitogen-activated protein kinase: preclinical-to-clinical translation. J Pharmacol Exp Ther 2009

15 Smith SJ, Fenwick PS, Nicholson AG, et al. Inhibitory effect of p38 mitogen-activated protein kinase inhibitors on cytokine release from human macrophages. Br J Pharmacol 2006; 149:393-404.

16 Macnee W, Allan RJ, Jones I, et al. Efficacy and safety of the oral p38 inhibitor PH-797804 in chronic obstructive pulmonary disease: a randomised clinical trial. Thorax 2013;68:738-45.

17 Lemire BB, Debigaré R, Dubé $A$, et al. MAPK signaling in the quadriceps of patients with chronic obstructive pulmonary disease. J Appl Physiol 2012;113:159-66.

18 Riddoch-Contreras J, George T, Natanek SA, et al. p38 mitogen-activated protein kinase is not activated in the quadriceps of patients with stable chronic obstructive pulmonary disease. COPD 2012;9:142-50.

19 Abraham SM, Lawrence T, Kleiman A, et al. Anti-inflammatory effects of dexamethasone are partly dependent on induction of dual specificity phosphatase 1. J Exp Med 2006;203:1883-9. 